\title{
Identification and functional analysis of risk-related microRNAs for the prognosis of patients with bladder urothelial carcinoma
}

\author{
JI GAO ${ }^{1}$, HONGYAN LI ${ }^{1}$, LEI LIU ${ }^{2}$, LIDE SONG ${ }^{3}$, YANTING LV ${ }^{3}$ and YUPING HAN ${ }^{1}$ \\ Departments of ${ }^{1}$ Urology and ${ }^{2}$ Endocrinology, China-Japan Union Hospital of Jilin University, Changchun, \\ Jilin 130033; ${ }^{3}$ Department of Pathology, Zhuji People's Hospital, Zhuji, Zhejiang 311800, P.R. China
}

Received August 3, 2016; Accepted July 5, 2017

DOI: $10.3892 / \mathrm{ol} .2017 .7124$

\begin{abstract}
The aim of the present study was to investigate risk-related microRNAs (miRs) for bladder urothelial carcinoma (BUC) prognosis. Clinical and microRNA expression data downloaded from the Cancer Genome Atlas were utilized for survival analysis. Risk factor estimation was performed using Cox's proportional regression analysis. A microRNA-regulated target gene network was constructed and presented using Cytoscape. In addition, the Database for Annotation, Visualization and Integrated Discovery was used for Gene Ontology (GO) and Kyoto Encyclopedia of Genes and Genomes pathway enrichment, followed by protein-protein interaction (PPI) network analysis. Finally, the K-clique method was applied to analyze sub-pathways. A total of 16 significant microRNAs, including hsa-miR-3622a and hsa-miR-29a, were identified $(\mathrm{P}<0.05)$. Following Cox's proportional regression analysis, hsa-miR-29a was screened as a prognostic marker of BUC risk $(\mathrm{P}=0.0449)$. A regulation network of hsa-miR-29a comprising 417 target genes was constructed. These target genes were primarily enriched in GO terms, including collagen fibril organization, extracellular matrix (ECM) organization and pathways, such as focal adhesion $(\mathrm{P}<0.05)$. A PPI network including 197 genes and 510 interactions, was constructed. The top 21 genes in the network module were enriched in GO terms, including collagen fibril organization and pathways, such as ECM receptor interaction $(\mathrm{P}<0.05)$. Finally, 4 sub-pathways of cysteine and methionine metabolism, including paths 00270_4,00270_1,00270_2 and 00270_5, were obtained $(\mathrm{P}<0.01)$ and identified to be enriched
\end{abstract}

Correspondence to: Dr Yuping Han, Department of Urology, China-Japan Union Hospital of Jilin University, 126 Xiantai Street, Changchun, Jilin 130033, P.R. China

E-mail: yupinghanyph@163.com

Abbreviations: BUC, bladder urothelial carcinoma; EGF, epidermal growth factor; GO, Gene Ontology; PPI, protein-protein interaction

Key words: bladder urothelial carcinoma, microRNA, signaling pathway, prognosis through DNA (cytosine-5)-methyltransferase (DNMT)3A, $D N M T 3 B$, methionine adenosyltransferase $2 \alpha(M A T 2 A)$ and spermine synthase $(S M S)$. The identified microRNAs, particularly hsa-miR-29a and its 4 associated target genes DNMT3A, $D N M T 3 B, M A T 2 A$ and $S M S$, may participate in the prognostic risk mechanism of BUC.

\section{Introduction}

Bladder urothelial carcinoma (BUC), a malignancy of the genitourinary system, is one of the most common types of bladder cancer(1). At present, the risk factors of BUC primarily comprise smoking and contact with aromatic amine chemicals (1). BUC may be divided into two categories: Non-muscle- and muscle-invasive BUC (2). Transurethral resection and radical cystectomy are the current treatment strategies for non-muscleand muscle-invasive BUC, respectively (3). Although numerous methods have been suggested, an effective treatment remains elusive due to high recurrence rates. A more thorough understanding of the underlying molecular mechanism of prognostic risk may be beneficial for the development of therapeutic interventions, and therefore the prognosis of patients with BUC.

MicroRNAs are a group of non-coding small RNAs, comprising $\sim 21$ nucleotides, which regulate the expression of target genes through binding to 3'-untranslated regions (UTRs) (4). Previous studies have demonstrated the association between microRNAs and risk factors in the prognosis of BUC (5), including miR-141 expression, which was revealed to be significantly downregulated in invasive bladder cancer (6). miR-141 regulates kelch-like ECH-associated protein 1 and controls the oxidative stress response that is associated with the prognosis of BUC (7). In addition, miR-205 targets $\mathrm{PH}$ domain leucine-rich repeat-containing protein phosphatase 2 and phosphatase and tensin homolog (PTEN), further influencing protein kinase B signaling (8). Cathomas et al (9) demonstrated that the expression of PTEN was associated with the development of chemotherapy- and castration-resistant cancer, as well as patient prognosis. Additionally, members of the epidermal growth factor (EGF) family have been suggested as potential prognostic markers in BUC (10); at the same time, resistance of EGF receptor is reversed by miR-200 in BUC (11). Therefore, miR-200 serves an important role in the prognostic risk of BUC and is an independent marker associated with an increased risk of non-muscle-invasive bladder cancer recurrence (12). 
An improved understanding of microRNA-associated risk factors may clarify the prognostic molecular mechanism of BUC. In the present study, microRNA expression profile data and clinical data were downloaded, survival curves were created to estimate risk factors and target genes regulated by microRNA were analyzed. In addition, regulation networks were constructed and functional analysis of target genes was performed. Finally, a protein-protein interaction (PPI) network of target genes regulated by microRNA was analyzed and a sub-pathway analysis was performed.

\section{Materials and methods}

Data sources. Clinical case data and expression profile data of microRNAs were downloaded from the Cancer Genome Atlas (TCGA; cancergenome.nih.gov) database on the BCGSC IlluminaHiSeq_miRNASeq platform (Canada's Michael Smith Genome Sciences Centre, Vancouver, BC, Canada). The TCGA microRNA expression data were obtained from 529 patients with BUC (download cut-off date, August 11, 2014). Reads per kilobase of exon per million mapped reads (RPKM) was used to quantify the expression value of patient microRNA (13) using the following formula: RPKM = total microRNA reads/[total mapped reads (million) x microRNA sequence length $(\mathrm{kb})]$. Additionally, clinical case data comprised 411 patients with urothelial bladder carcinoma (download cut-off date, August 11, 2014). A total of 408 cases that exhibited microRNA expression profile data were selected for analysis.

Survival analysis. The mean expression value of each microRNA in the 408 cases was calculated as the critical value. All cases were divided into two groups: MicroRNA expression greater than the critical value, and microRNA expression equal to or less than the critical value of microRNA expression. A Kaplan-Meier estimator survival curve was created for microRNA in the two groups and a log-rank test was applied to analyze the significance. MicroRNAs exhibiting a significantly different survival curve were screened as candidates for prognostic factors. $\mathrm{P}<0.05$ was considered to indicate a statistically significant difference.

Identification of risk-related miRNAs. Cox's proportional hazards regression model was used to estimate the risk factors for collected clinical data and microRNA that demonstrated a significant effect on the survival curves. KMsurv (14) and survival (15) packages in R language were applied for the plotting of survival curves and Cox's proportional hazards regression model. Cox's proportional hazards regression model was created according to the backward selection method; variables were first introduced and subsequently the free variables with no significant differences were eliminated [hazard ratio (HR), 0.99997; $\mathrm{P}=0.0449]$.

Analysis of key target genes regulated by microRNA. MicroRNA target genes were predicted from relevant databases, including two validation databases, miRNecords (16) and miRWalk (17). To be applicable for the present study, the predicted regulatory association must have existed in at least three of the following databases: miRanda (18),
mirTarget2 (19), PicTar (20), PITA (21) and TargetScan (22). Genes that complied with the two aforementioned requirements were screened. A regulatory network was created and visualized using Cytoscape (23), based on the predicted target genes. Cytoscape is an open source software platform for visualizing complex networks and integrating these with any data type.

Functional analysis of target genes. The Database for Annotation, Visualization and Integrated Discovery, which provides analytical tools for extracting biological relevance from collections of genes (24), was used for Gene Ontology (GO) and Kyoto Encyclopedia of Genes and Genomes pathway enrichment analysis of target genes in the microRNA-regulated network. $\mathrm{P}<0.05$ was used as the threshold criterion.

PPI network analysis of microRNA target genes. The PPI network of target genes was constructed using the Search Tool for Retrieval of Interacting Genes database, which provided integrated knowledge of the known and predicted associations for protein networks (25). PPI pairs with a combined score $>0.4$ were screened and visualized using Cytoscape.

Sub-pathway analysis of target genes. The K-clique method was used to divide metabolic pathways into sub-pathways, based on structural information, and to identify risk pathways using hypergeometric test (26). ISubpathway Miner limma (27) in $\mathrm{R}$ was applied for investigation of the processes of K-clique recognized risk sub-pathways. Sub-pathways with $\mathrm{P}<0.05$ were considered to be risk sub-pathways. The associations between pathways and disease with target gene involvement were investigated.

\section{Results}

Survival analysis. A total of 16 survival curves that significantly affected microRNA were obtained. Among them, the survival curves, including those for hsa-miR-3622a, hsa-miR-1292 and hsa-miR-3138 with significantly longer survival times and has-miR-29a with shorter survival time, were obtained on the condition that expression of microRNA was higher than the mean critical value. Another 12 survival curves exhibited significant longer survival time on the condition that the expression of microRNA was lower than mean value.

Cox's proportional regression analysis. Prognostic hazard ratios of microRNA were obtained using Cox's proportional regression analysis of the aforementioned 16 microRNA expression values. hsa-miR-29a was identified as a risk microRNA associated with the prognosis of UBC.

Risk-related microRNA regulation network. A regulation network of hsa-miR-29a was constructed by collecting and arranging database data of microRNA regulated target genes; a total 417 target genes were contained in the network (Fig. 1).

Functional enrichment analysis of target genes. Based on the results of enrichment analysis, the target genes of hsa-miR-29a were primarily enriched in GO terms, including collagen fibril 


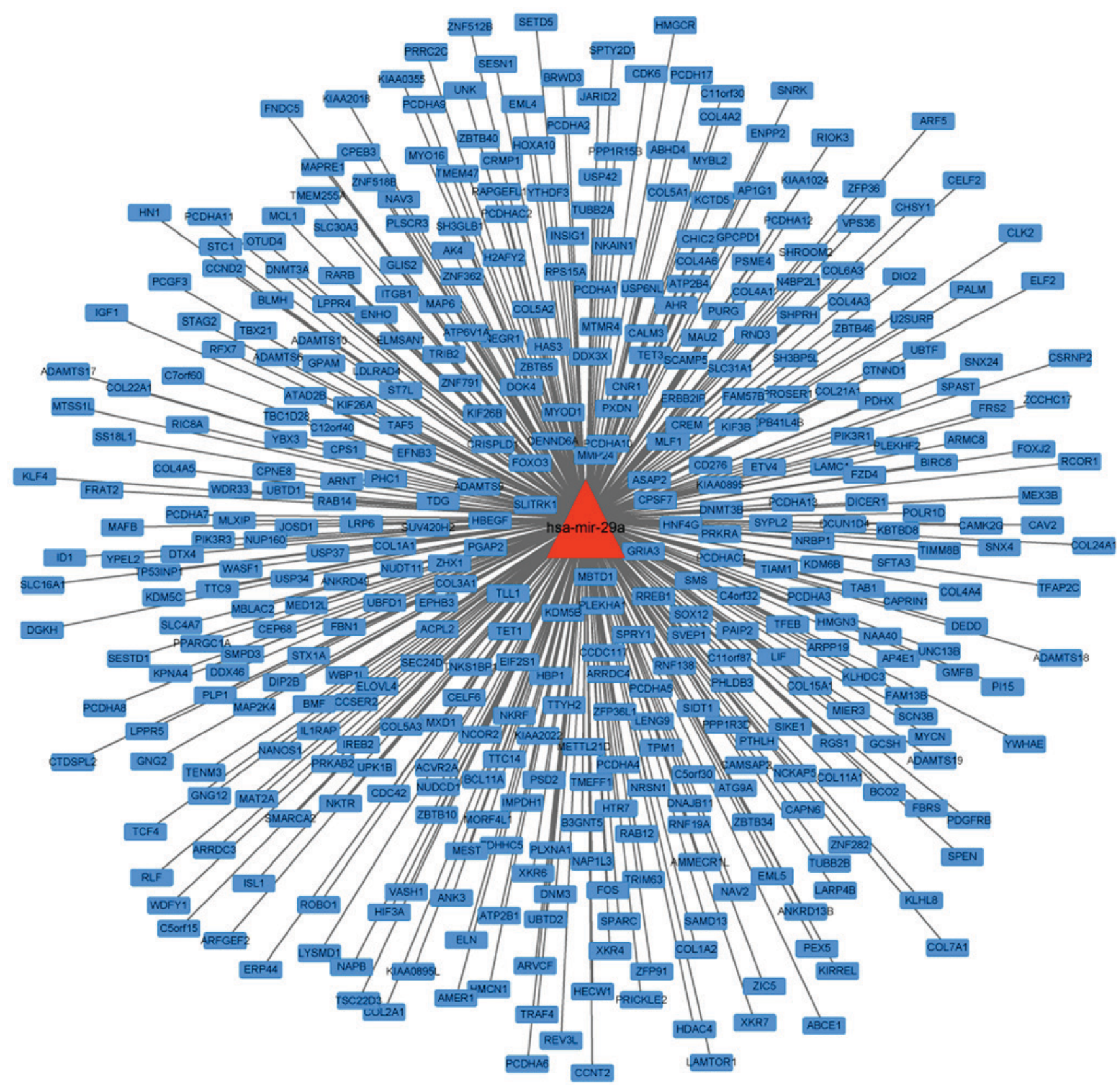

Figure 1. Regulatory network constructed using the risk microRNA, miR-29a, and its regulated target genes. The red triangle represents microRNA, and the blue rectangles represent target genes. miR, microRNA.

organization $\left(\mathrm{P}=2.64 \times 10^{-6}\right)$, extracellular matrix $(\mathrm{ECM})$ organization $\left(\mathrm{P}=2.02 \times 10^{-5}\right)$, homophilic cell adhesion $\left(\mathrm{P}=3.66 \times 10^{-5}\right)$ and extracellular structure organization $\left(\mathrm{P}=7.45 \times 10^{-5}\right)$. These target genes were also enriched in pathways that included focal adhesion $\left(\mathrm{P}=5.06 \times 10^{-10}\right)$, ECM-receptor interaction $\left(\mathrm{P}=1.16 \times 10^{-9}\right)$ and small cell lung cancer $\left(\mathrm{P}=7.71 \times 10^{-6}\right)$, and pathways in cancer $\left(\mathrm{P}=1.11 \times 10^{-3}\right)$ (Table I).

PPI network analysis of target genes. A PPI network with 197 genes and 510 edges was constructed (Fig. 2). In this network, collagen type $1 \alpha$ chain 2 (COL1A2)2, COL1A1 and COL3Al were the top three nodes, the degrees of which were 25,24 and 24, respectively. In addition, the top 5 pairs with the greatest combined score were phosphatidylinositol 3-kinase regulatory subunit 1-platelet-derived growth factor receptor $\beta$ (0.999), COL5A2-COL5A1 (0.992), L5A2-COL11A1 (0.999),
L5A1-COL5A3 (0.999) and COL4A6-COL4A5 (0.999). Values in brackets are the combined score value.

Furthermore, a network module with 21 genes was screened from the PPI network (Fig. 3). The enrichment results of this module are presented in Table II. The genes in this module were primarily enriched in functions that included collagen fibril organization $\left(\mathrm{P}=2.97 \times 10^{-15}\right)$, ECM organization $\left(\mathrm{P}=3.01 \times 10^{-15}\right)$, cell adhesion $\left(\mathrm{P}=1.15 \times 10^{-13}\right)$ and biological adhesion $\left(\mathrm{P}=1.17 \times 10^{-13}\right)$.

Risk sub-pathway analysis. A total of 4 sub-pathways of cysteine and methionine metabolism were obtained, including paths 00270_4 ( $\left.\mathrm{P}=4.11 \times 10^{-4}\right), 00270 \_1\left(\mathrm{P}=6.16 \times 10^{-4}\right), 00270 \_2$ $\left(\mathrm{P}=5.40 \times 10^{-3}\right)$ and 00270_5 $\left(\mathrm{P}=6.26 \times 10^{-3}\right)$. Paths 00270_4 and 00270_1 were enriched by DNA (cytosine-5)-methyltransferase $3 \alpha$ (DNMT3A), DNMT3 $\beta$ (DNMT3B), methionine 
Table I. Top 5 GO terms and pathways enrichment of hsa-miR-29a target genes.

\begin{tabular}{|c|c|c|c|}
\hline Category & Term & Count & P-value \\
\hline GOTERM_BP_FAT & GO:0030199 collagen fibril organization & 8 & $2.64 \times 10^{-6}$ \\
\hline GOTERM_BP_FAT & GO:0030198 ECM organization & 12 & $2.02 \times 10^{-5}$ \\
\hline GOTERM_BP_FAT & GO:0007156 homophilic cell adhesion & 13 & $3.66 \times 10^{-5}$ \\
\hline GOTERM_BP_FAT & GO:0043062 extracellular structure organization & 14 & $7.45 \times 10^{-5}$ \\
\hline GOTERM_BP_FAT & GO:0022610 biological adhesion & 33 & $9.53 \times 10^{-5}$ \\
\hline GOTERM_CC_FAT & GO:0005581 collagen & 18 & $4.91 \times 10^{-20}$ \\
\hline GOTERM_CC_FAT & GO:0044420 ECM part & 24 & $1.84 \times 10^{-16}$ \\
\hline GOTERM_CC_FAT & GO:0005578 proteinaceous ECM & 34 & $2.30 \times 10^{-14}$ \\
\hline GOTERM_CC_FAT & GO:0031012 ECM & 35 & $3.42 \times 10^{-14}$ \\
\hline GOTERM_CC_FAT & GO:0005604 basement membrane & 15 & $6.33 \times 10^{-10}$ \\
\hline GOTERM_MF_FAT & GO:0005201 ECM structural constituent & 19 & $9.83 \times 10^{-13}$ \\
\hline GOTERM_MF_FAT & GO:0048407 PDGF binding & 7 & $6.57 \times 10^{-8}$ \\
\hline GOTERM_MF_FAT & GO:0005198 structural molecule activity & 30 & $4.42 \times 10^{-4}$ \\
\hline GOTERM_MF_FAT & GO:0003677 DNA binding & 76 & $1.83 \times 10^{-3}$ \\
\hline GOTERM_MF_FAT & GO:0019838 growth factor binding & 9 & $3.22 \times 10^{-3}$ \\
\hline KEGG_PATHWAY & hsa04510: Focal adhesion & 22 & $5.06 \times 10^{-10}$ \\
\hline KEGG_PATHWAY & hsa04512: ECM-receptor interaction & 15 & $1.16 \times 10^{-9}$ \\
\hline KEGG_PATHWAY & hsa05222: Small cell lung cancer & 11 & $7.71 \times 10^{-6}$ \\
\hline KEGG_PATHWAY & hsa05200: Pathways in cancer & 17 & $1.11 \times 10^{-3}$ \\
\hline KEGG_PATHWAY & hsa05214: Glioma & 7 & $1.85 \times 10^{-3}$ \\
\hline REACTOME_PATHWAY & REACT_16888: Signaling by PDGF & 14 & $2.20 \times 10^{-10}$ \\
\hline REACTOME_PATHWAY & REACT_18266: Axon guidance & 12 & $1.99 \times 10^{-9}$ \\
\hline REACTOME_PATHWAY & REACT_13552: Integrin cell surface interactions & 11 & $4.20 \times 10^{-6}$ \\
\hline REACTOME_PATHWAY & REACT_604: Hemostasis & 10 & $4.97 \times 10^{-2}$ \\
\hline
\end{tabular}

ECM, extracellular matrix; PDGF, platelet-derived growth factor.

Table II. Top 5 GO terms and pathway enrichment of hsa-miR-29a target genes in network module 1.

\begin{tabular}{|c|c|c|c|}
\hline Category & Term & Count & P-value \\
\hline GOTERM_BP_FAT & GO:0030199 collagen fibril organization & 8 & $2.97 \times 10^{-15}$ \\
\hline GOTERM_BP_FAT & GO:0030198 ECM organization & 10 & $3.01 \times 10^{-15}$ \\
\hline GOTERM_BP_FAT & GO:0007155 cell adhesion & 14 & $1.15 \times 10^{-13}$ \\
\hline GOTERM_BP_FAT & GO:0022610 biological adhesion & 14 & $1.17 \times 10^{-13}$ \\
\hline GOTERM_BP_FAT & GO:0043062 extracellular structure organization & 10 & $1.90 \times 10^{-13}$ \\
\hline GOTERM_CC_FAT & GO:0005581 collagen & 18 & $2.85 \times 10^{-43}$ \\
\hline GOTERM_CC_FAT & GO:0044420 ECM part & 18 & $7.38 \times 10^{-33}$ \\
\hline GOTERM_CC_FAT & GO:0005578 proteinaceous ECM & 20 & $4.29 \times 10^{-30}$ \\
\hline GOTERM_CC_FAT & GO:0031012 ECM & 20 & $1.86 \times 10^{-29}$ \\
\hline GOTERM_CC_FAT & GO:0044421 extracellular region part & 20 & $6.82 \times 10^{-21}$ \\
\hline GOTERM_MF_FAT & GO:0005201 ECM structural constituent & 16 & $8.63 \times 10^{-30}$ \\
\hline GOTERM_MF_FAT & GO:0005198 structural molecule activity & 18 & $4.09 \times 10^{-20}$ \\
\hline GOTERM_MF_FAT & GO:0048407 PDGF binding & 6 & $2.32 \times 10^{-12}$ \\
\hline GOTERM_MF_FAT & GO:0019838 growth factor binding & 6 & $4.43 \times 10^{-7}$ \\
\hline GOTERM_MF_FAT & GO:0005178 integrin binding & 4 & $9.62 \times 10^{-5}$ \\
\hline KEGG_PATHWAY & hsa04512: ECM-receptor interaction & 14 & $2.60 \times 10^{-24}$ \\
\hline KEGG_PATHWAY & hsa04510: Focal adhesion & 14 & $3.93 \times 10^{-19}$ \\
\hline KEGG_PATHWAY & hsa05222: Small cell lung cancer & 5 & $4.42 \times 10^{-5}$ \\
\hline KEGG_PATHWAY & hsa05200: Pathways in cancer & 5 & $7.63 \times 10^{-3}$ \\
\hline REACTOME_PATHWAY & REACT_18266: Axon guidance & 12 & $2.01 \times 10^{-20}$ \\
\hline REACTOME_PATHWAY & REACT_16888: Signaling by PDGF & 12 & $5.11 \times 10^{-19}$ \\
\hline REACTOME_PATHWAY & REACT_13552: Integrin cell surface interactions & 9 & $3.37 \times 10^{-11}$ \\
\hline
\end{tabular}

ECM, extracellular matrix; PDGF, platelet-derived growth factor. 
Table III. Analyzed results of risk pathways.

\begin{tabular}{llll}
\hline Pathway ID & \multicolumn{1}{c}{ Pathway name } & P-value & Gene \\
\hline path:00270_4 & Cysteine and methionine metabolism & $4.11 \times 10^{-4}$ & DNMT3A,DNMT3B,MAT2A,SMS \\
path:00270_1 & Cysteine and methionine metabolism & $6.16 \times 10^{-4}$ & DNMT3A,DNMT3B, MAT2A,SMS \\
path:00270_2 & Cysteine and methionine metabolism & $5.40 \times 10^{-3}$ & DNMT3A,DNMT3B, MAT2A \\
path:00270_5 & Cysteine and methionine metabolism & $6.26 \times 10^{-3}$ & DNMT3A,DNMT3B, MAT2A \\
\hline
\end{tabular}

DNMT, DNA(cytosine-5)-methyltransferase; MAT, methionine adenosyltransferase; SMS, spermine synthase.

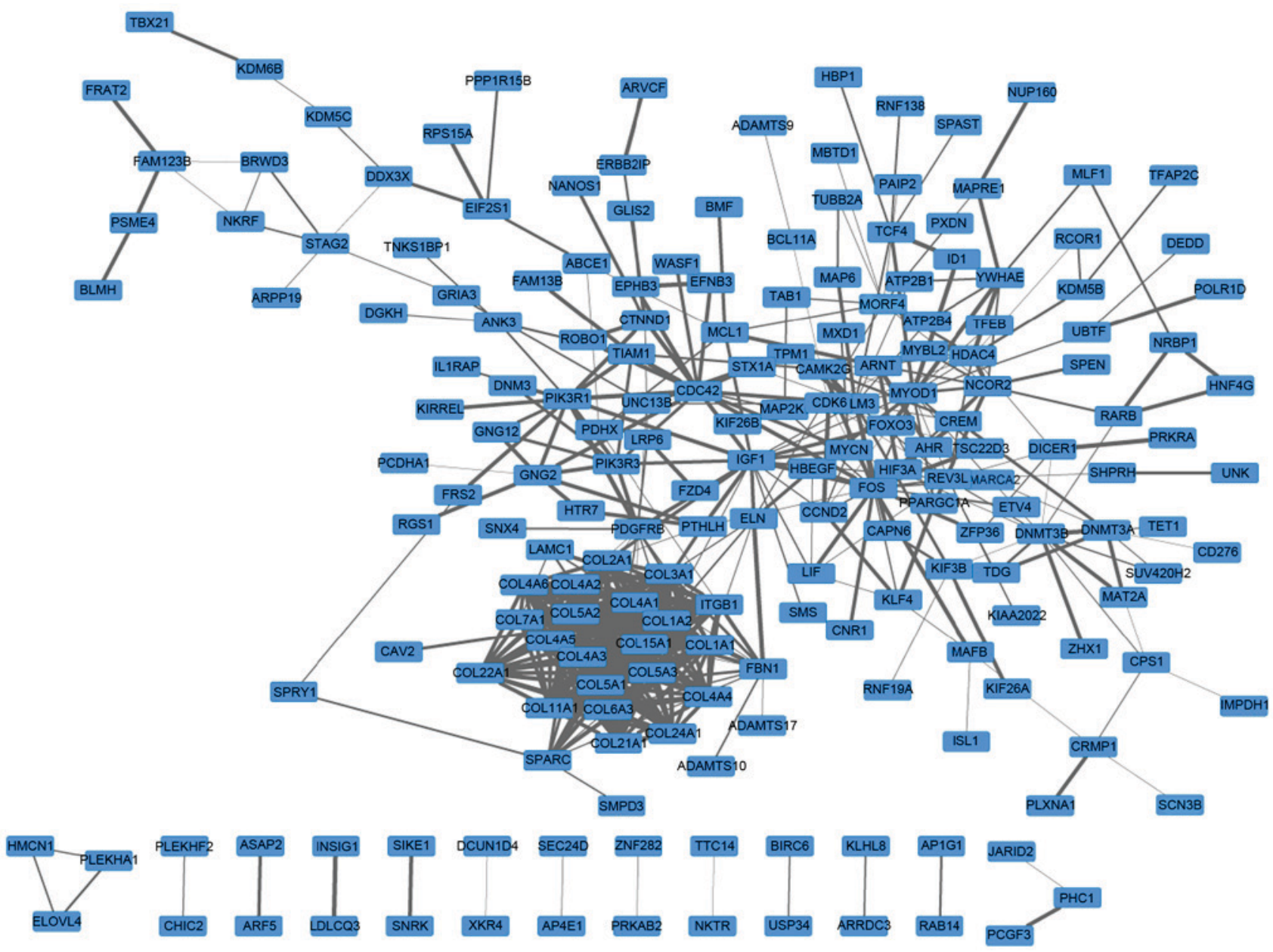

Figure 2. PPI network constructed using the target genes of miR-29a. Blue rectangles represent target genes of miR-29a and edges represent interactions between target genes. The thickness of each edge is in proportion to the combined score.

adenosyltransferase $2 \alpha(M A T 2 A)$ and spermine synthase $(S M S)$, whereas paths 00270_2 and 00270_5 were enriched by DNMT3A, DNMT3B and MAT2A (Table III).

\section{Discussion}

BUC is a malignancy of the genitourinary system that is difficult to effectively treat due to high recurrence rates (28). In the present study, hsa-miR-29a was screened as a prognostic risk-related microRNA of BUC. In addition, 21 genes in the network module were enriched in GO terms, including collagen fibril organization and ECM organization, and were enriched in pathways, including ECM-receptor interaction and focal adhesion. Finally, 4 pathways, including path00270_4, path00270_1, path00270_2 and path00270_5, were obtained and enriched by 4 target genes, DNMT3A DNMT3B, MAT2A and $S M S$.

hsa-miR-29a was the only microRNA that significantly affected the prognosis of BUC. hsa-miR-29a is a microRNA member of the miR-29 family, the dysregulation of which has been demonstrated to affect DNMT3A expression in the HL1 cell line (29). Notably, in the DNMT3A mutation samples, DNA methylation patterns were altered (30). In other types of cancer, including lung cancer, the miR-29 family reversed biological processes of aberrant DNA methylation and was associated with a poor prognosis in cancer (31). In addition, 


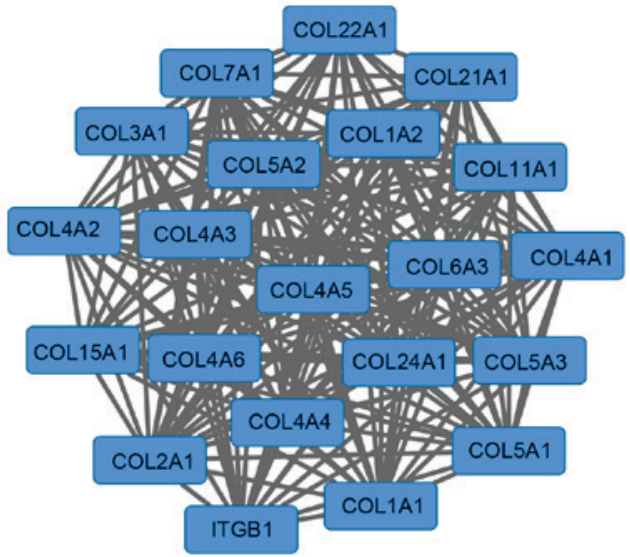

Figure 3. Network module 1 extracted from the PPI network. Blue rectangles represent target genes and edges represent interactions between target genes.

downregulated miR-29a may promote transforming growth factor $\beta$ induction and further promote the fibrotic response by interacting with genes, including fibrillin, elastin and collagens (32). Similar to DNMT3A, DNMT3B also exhibits complementarities with the miR-29 family at 3'-UTRs (31). The synthesis of S-adenosyl-(L)-methionine (adoMet), the primary methyl group donor in humans, is the primary step in the process of methionine metabolism (33). Through Adohcy, the transfer of activated methyl groups is naturally catalyzed from AdoMet to C5 atom by DNMT3A and DNMT3B (34). Consistent with previous studies, results in the present study revealed that $D N M T 3 A$ and $D N M T 3 B$ were regulated by miR-29a, and enriched in the cysteine and methionine metabolism pathway, affecting the prognosis of $B U C$.

Furthermore, miR-29a has also been demonstrated to regulate MAT2A and SMS.MAT2A is a mammalian gene that encodes MAT (35). AdoMet is an intermediate metabolite that also functions as an intracellular control switch, which regulates essential functions (36). Furthermore, MAT2A serves a role in the methionine cycle pathway, which is an important metabolic pathway (37). Although the molecular mechanisms of SMS associated with BUC prognostic risk have not been reported, the results of the present study suggest that they may serve important roles in BUC prognostic risk through their involvement in the cysteine and methionine metabolism pathway.

In addition to the aforementioned pathways, miR-29a was also enriched in ECM organization and biological adhesion. Ioachim et al (38) demonstrated that thrombospondin type 1 serves an important role in the prognosis of cancer, being enriched in ECM organization pathways. $\beta 1$-integrin has been demonstrated to downregulate expression of miR-29a, whilst increased expression of $\beta 1$-integrin in BUC cells induces tissue invasion (39). Cell invasion is the primary factor associated with poor prognosis (40). Through these pathways, miR-29a may exhibit an important prognostic risk.

Although several key genes and pathways associated with BUC were identified using comprehensive bioinformatic methods, no experiment was conducted to verify the results and this therefore presents a clear limitation to the present study. Further experimental studies of diverse samples are thus required to validate the results of the present study.
In conclusion, the identified microRNAs, particularly hsa-miR-29a, may serve important roles in the prognostic risk mechanism of BUC through the regulation of 4 target genes, including DNMT3A, DNMT3B, MAT2A and SMS, and through involvement in cysteine and methionine metabolism pathways. However, further study is required to support the potential association between microRNAs, target genes and prognostic risk factors.

\section{References}

1. Ploeg M, Aben KK and Kiemeney LA: The present and future burden of urinary bladder cancer in the world. World J Urol 27: 289-293, 2009.

2. Kauffman EC, Ng CK, Lee MM, Otto BJ, Wang GJ and Scherr DS: Early oncological outcomes for bladder urothelial carcinoma patients treated with robotic-assisted radical cystectomy. BJU Int 107: 628-635, 2011.

3. Wright JL, Black PC, Brown GA, Porter MP, Kamat AM, Dinney CP and Lin DW: Differences in survival among patients with sarcomatoid carcinoma, carcinosarcoma and urothelial carcinoma of the bladder. J Urol 178: 2302-2307, 2007.

4. Bartel DP: MicroRNAs: Genomics, biogenesis, mechanism, and function. Cell 116: 281-297, 2004.

5. Shi Z, Wei Q, Zhang M and She J: MicroRNAs in bladder cancer: Expression profiles, biological functions, regulation, and clinical implications. Crit Rev Eukaryot Gene Expr 24: 55-75, 2014.

6. Wszolek MF,Rieger-Christ KM,Kenney PA, Gould JJ, Silva Neto B, Lavoie AK, Logvinenko T, Libertino JA and Summerhayes IC: A MicroRNA expression profile defining the invasive bladder tumor phenotype. Urol Oncol 29: 794-801.e1, 2011.

7. Soini Y, Haapasaari KM, Vaarala MH, Turpeenniemi-Hujanen T, Kärjä V and Karihtala P: 8-hydroxydeguanosine and nitrotyrosine are prognostic factors in urinary bladder carcinoma. Int $\mathrm{J}$ Clin Exp Pathol 4: 267-275, 2011.

8. Cai J, Fang L, Huang Y, Li R, Yuan J, Yang Y, Zhu X, Chen B, Wu J and Li M: miR-205 targets PTEN and PHLPP2 to augment AKT signaling and drive malignant phenotypes in non-small cell lung cancer. Cancer Res 73: 5402-5415, 2013.

9. Cathomas R, Rothermundt C, Klingbiel D, Bubendorf L, Jaggi R, Betticher DC, Brauchli P, Cotting D, Droege C, Winterhalder R, et al: Efficacy of cetuximab in metastatic castration-resistant prostate cancer might depend on EGFR and PTEN expression: Results from a phase II trial (SAKK 08/07). Clin Cancer Res 18: 6049-6057, 2012.

10. Thogersen VB, Sørensen BS, Poulsen SS, Orntoft TF, Wolf H and Nexo E: A subclass of HER1 ligands are prognostic markers for survival in bladder cancer patients. Cancer Res 61: 6227-6233, 2001.

11. Adam L, Zhong M, Choi W, Qi W, Nicoloso M, Arora A, Calin G, Wang H, Siefker-Radtke A, McConkey D, et al: miR-200 expression regulates epithelial-to-mesenchymal transition in bladder cancer cells and reverses resistance to epidermal growth factor receptor therapy. Clin Cancer Res 15: 5060-5072, 2009.

12. Yun SJ, Jeong P, Kim WT, Kim TH, Lee YS, Song PH, Choi YH, Kim IY, Moon SK and Kim WJ: Cell-free microRNAs in urine as diagnostic and prognostic biomarkers of bladder cancer. Int $\mathrm{J}$ Oncol 41: 1871-1878, 2012.

13. Trapnell C, Pachter L and Salzberg SL: TopHat: Discovering splice junctions with RNA-Seq. Bioinformatics 25: 1105-1111, 2009.

14. Diez D: Survival analysis in R, 2012. https://folk.ntnu.no/bo/ TMA4275/Download/R.tutorialDiez.pdf. Accessed August 11, 2014.

15. Therneau TM and Grambsch PM: Modeling survival data: Extending the Cox model. Springer-Verlag, New York, NY, 2000.

16. Xiao F, Zuo Z, Cai G, Kang S, Gao X and Li T: miRecords: An integrated resource for microRNA-target interactions. Nucleic Acids Res 37: D105-D110, 2009.

17. Dweep H, Sticht C, Pandey P and Gretz N: miRWalk-database: Prediction of possible miRNA binding sites by 'walking' the genes of three genomes. J Biomed Inform 44: 839-847, 2011.

18. Enright AJ, John B, Gaul U, Tuschl T, Sander C and Marks DS: MicroRNA targets in Drosophila. Genome Biol 5: R1, 2003.

19. Wang $X$ and El Naqa IM: Prediction of both conserved and nonconserved microRNA targets in animals. Bioinformatics 24: 325-332, 2008 . 
20. Krek A, Grün D, Poy MN, Wolf R, Rosenberg L, Epstein EJ, MacMenamin P, da Piedade I, Gunsalus KC, Stoffel M and Rajewsky N: Combinatorial microRNA target predictions. Nat Genet 37: 495-500, 2005.

21. Kertesz M, Iovino N, Unnerstall U, Gaul U and Segal E: The role of site accessibility in microRNA target recognition. Nat Genet 39: 1278-1284, 2007.

22. Lewis BP, Shih IH, Jones-Rhoades MW, Bartel DP and Burge CB: Prediction of mammalian microRNA targets. Cell 115: 787-798, 2003.

23. Shannon P, Markiel A, Ozier O, Baliga NS, Wang JT, Ramage D, Amin N, Schwikowski B and Ideker T: Cytoscape: A software environment for integrated models of biomolecular interaction networks. Genome Res 13: 2498-2504, 2003.

24. Huang da W, Sherman BT and Lempicki RA: Systematic and integrative analysis of large gene lists using DAVID bioinformatics resources. Nat Protoc 4: 44-57, 2009.

25. Franceschini A, Szklarczyk D, Frankild S, Kuhn M, Simonovic M, Roth A, Lin J, Minguez P, Bork P, von Mering C and Jensen LJ STRING v9.1: Protein-protein interaction networks, with increased coverage and integration. Nucleic Acids Res 41: D808-D815, 2013.

26. Toivonen R, Onnela JP, Saramäki J, Hyvönen J and Kaski K: A model for social networks. Physica A: Statistical Mechanics and its. Applications 371: 851-860, 2006

27. Li C and Li MC: Package 'iSubpathwayMiner', 2013. http://ftp. cs.pu.edu.tw/network/CRAN/web/packages/iSubpathwayMiner/ iSubpathwayMiner.pdf. Accessed August 11, 2014.

28. Schmidbauer J, Witjes F, Schmeller N, Donat R, Susani M and Marberger M; Hexvix PCB301/01 Study Group: Improved detection of urothelial carcinoma in situ with hexaminolevulinate fluorescence cystoscopy. J Urol 171: 135-138, 2004.

29. Iorio MV and Croce CM: MicroRNA dysregulation in cancer: Diagnostics, monitoring and therapeutics. A comprehensive review. EMBO Mol Med 4: 143-159, 2012.

30. Yan XJ, Xu J, Gu ZH, Pan CM, Lu G, Shen Y, Shi JY, Zhu YM, Tang L, Zhang XW, et al: Exome sequencing identifies somatic mutations of DNA methyltransferase gene DNMT3A in acute monocytic leukemia. Nat Genet 43: 309-315, 2011.

31. Fabbri M, Garzon R, Cimmino A, Liu Z, Zanesi N, Callegari E, Liu S, Alder $\mathrm{H}$, Costinean S, Fernandez-Cymering C, et al: MicroRNA-29 family reverts aberrant methylation in lung cancer by targeting DNA methyltransferases 3A and 3B. Proc Natl Acad Sci USA 104: 15805-15810, 2007.
32. van Rooij E, Sutherland LB, Thatcher JE, DiMaio JM, Naseem RH, Marshall WS, Hill JA and Olson EN: Dysregulation of microRNAs after myocardial infarction reveals a role of miR-29 in cardiac fibrosis. Proc Natl Acad Sci USA 105: 13027-13032, 2008.

33. Pradhan S, Bacolla A, Wells RD and Roberts RJ: Recombinant human DNA (cytosine-5) methyltransferase. I. Expression, purification, and comparison of de novo and maintenance methylation. J Biol Chem 274: 33002-33010, 1999.

34. Gowher H and Jeltsch A: Enzymatic properties of recombinant Dnmt3a DNA methyltransferase from mouse: The enzyme modifies DNA in a non-processive manner and also methylates non-CpG [correction of non-CpA] sites. J Mol Biol 309: 1201-1208, 2001.

35. Mato JM, Corrales FJ, Lu SC and Avila MA: S-Adenosylmethionine: A control switch that regulates liver function. FASEB J 16: 15-26, 2002.

36. Roje S: S-Adenosyl-L-methionine: Beyond the universal methyl group donor. Phytochemistry 67: 1686-1698, 2006.

37. Main PA, Angley MT, Thomas P, O'Doherty CE and Fenech M Folate and methionine metabolism in autism: A systematic review. Am J Clin Nutr 91: 1598-1620, 2010.

38. Ioachim E, Michael MC, Salmas M, Damala K, Tsanou E, Michael MM, Malamou-Mitsi V and Stavropoulos NE: Thrombospondin-1 expression in urothelial carcinoma: Prognostic significance and association with p53 alterations, tumour angiogenesis and extracellular matrix components. BMC Cancer 6: 140, 2006.

39. Chakraborty A, White SM and Lerner SP: Granulocyte colonystimulating factor receptor signals for beta1-integrin expression and adhesion in bladder cancer. Urology 63: 177-183, 2004.

40. Lopez-Beltran A and Cheng L: Histologic variants of urothelial carcinoma: Differential diagnosis and clinical implications. Hum Pathol 37: 1371-1388, 2006. 